\title{
Tese por compêndio de doutorado em ciências sociais e educação: Vantagens, riscos e propostas
}

Thesis by compendium in doctoral programs in social sciences and education:
Advantages, risks and proposals
Tesis por compendio en doctorados en ciencias sociales y educación:
Ventajas, riesgos y propuestas

RENATO GAZMURI STEIN

https://orcid.org/0000-0002-0927-875X

Universidad Diego Portales, Chile

Faculdade de Educação

Departamento de Ciências Sociais

Santiago, Chile

ALEJANDRA FALABELLA AMBROSIO

Universidad Alberto Hurtado, Chile Orcid Id: 0000-0003-2755-4911

Correo: afalabel@uahurtado.cl

TAMARA CANESSA AGUILAR

Universidad Diego Portales, Chile

Orcid Id: 0000-0002-0997-1562

Correo: tamara.canessa@gmail.com

\begin{abstract}
Resumo: O objetivo deste artigo é analisar as vantagens e os riscos da implementação de Teses por Compêndio de Publicações (TCP) em programas de doutorado em ciências sociais e de educação. Para isso, foram comparados os requisitos e características dos TCPs em 13 programas de doutorado chilenos e internacionais, e foram realizadas quatro entrevistas em profundidade com alunos que fizeram ou estão fazendo suas teses neste formato. Por fim, apresentamos um conjunto de propostas ou critérios a ter em consideração na implementação da opção TCP, com o propósito de salvaguardar a qualidade, pertinência e diversidade dos processos de formação dos estudantes.
\end{abstract}

Palavras-chave: Tese por Compêndio de Publicações / Doutorados / Formação doutoral.

Abstract: The purpose of this article is to analyze the advantages and risks of the implementation of Thesis by Compendium of Publications (TCP) in doctoral programs in social sciences and education. For this, the requirements and characteristics of the TCPs were compared in 13 Chilean and international doctoral programs, and four in-depth interviews were conducted with students who did or are doing their theses in this format. Finally, a set of proposals or criteria to consider when implementing the TCP option is postulated, to safeguard the quality, relevance, and diversity of the students' training processes.

Keywords: Thesis by Compendium of Publications / Doctorates / Doctoral training. 
Resumen: Elpropósito de este artículo es analizar las ventajas y riesgos de la implementación de Tesis por Compendio de Publicaciones (TCP) en programas doctorales en ciencias sociales y educación. Para eso, se compararon los requisitos y características de las TCP en 13 programas doctorales, chilenos e internacionales, y se realizaron cuatro entrevistas en profundidad a estudiantes que bicieron o están realizando sus tesis en este formato. Finalmente se postula un conjunto de propuestas o criterios a considerar al implementar la opción TCP, con el propósito de resguardar la calidad, pertinencia y diversidad de los procesos formativos de los estudiantes.

Palabras clave: Tesis por Compendio de Publicaciones/ Doctorados/ Formación doctoral.

\section{INTRODUCCIÓN}

La labor académica y el modo de producir conocimiento científico en ciencias sociales y educación han cambiado de forma radical en las últimas décadas. Ello ha significado, en consecuencia, que el modo de formar doctores en estas áreas del conocimiento también ha vivido transformaciones importantes.

Una de estas transformaciones afecta al corazón de los estudios doctorales: la realización de la tesis. Como alternativa a la tradicional tesis monográfica, diversos programas alrededor del mundo han comenzado a introducir la modalidad de Tesis por Compendio de Publicaciones (TCP), es decir, un conjunto de artículos científicos organizados en un compendio. Aunque este cambio pudiese parecer una respuesta natural a las exigencias contemporáneas, no es un cambio inocuo. Es un cambio profundo que requiere ser examinado críticamente, con el fin de que los programas doctorales tomen decisiones con cautela, y consideren las distintas ventajas y riesgos de esta nueva modalidad.

Este estudio surge como una inquietud de la comunidad académica del Doctorado en Educación de las universidades Diego Portales y Alberto Hurtado. El propósito es identificar y describir las ventajas y riesgos de las TCP, y así también, realizar un conjunto de propuestas a tener en consideración para su implementación. Para eso se analizan los requisitos y características de 13 programas de doctorado en ciencias sociales y en educación, chilenos e internacionales (en Europa, Norte América y Oceanía), que han implementado TCP. Junto con eso, se realizan cuatro entrevistas en profundidad a estudiantes que cursaron o están cursando sus doctorados bajo esta modalidad, con el objetivo de describir las ventajas y riesgos de esta opción desde sus experiencias. El artículo concluye con un conjunto de propuestas para atender a los desafíos que supone la implementación de la opción de TCP, con el propósito de resguardar la calidad, pertinencia y diversidad de los procesos formativos de los estudiantes de doctorado. 


\section{LITERATURA DE REFERENCIA SOBRE LAS TESIS POR COMPENDIO DE PUBLICACIONES}

En las últimas décadas la concepción de la labor académica y los criterios según los cuales se valora la producción científica han cambiado como producto de la progresiva instalación de políticas de rendición de cuentas y el incentivo a la competencia entre instituciones de Educación Superior. De manera creciente, la calidad de las universidades y de sus académicos y académicas se miden en función de la productividad científica y su reflejo en indicadores bibliométricos (Fardella, Baleriola y Enciso, 2020; O’Keeffe, 2019; Mason, Merga y Morris, 2019).

Estas transformaciones han repercutido en la formación doctoral. La creciente competitividad del mercado académico y de la adjudicación de fondos concursables de investigación ha incentivado la necesidad, cada vez más apremiante, de que las y los estudiantes publiquen durante el desarrollo de sus doctorados (Peacock, 2017). En este contexto, el objetivo de la tesis doctoral también ha ido cambiando. Tradicionalmente estas tenían como objetivos preferentes la formación de investigadores e investigadoras a través del desarrollo de una primera investigación de envergadura y la certificación de la progresión del candidato al grado de doctor. La publicación de artículos, si bien era deseable, era concebida como un objetivo paralelo a la elaboración de la tesis. Hoy estos objetivos se han ido alineando, tomando cada vez más prioridad la necesidad de publicar artículos, idealmente en revistas de alta indexación (Rigby y Jones, 2020).

En gran medida como respuesta a esa necesidad, los programas doctorales en distintas disciplinas, y de manera creciente alrededor del mundo, han ido implementado progresivamente las TCP como una alternativa a las tesis monográficas. En este formato, las investigaciones doctorales se traducen en un conjunto de artículos independientes agrupados en un compendio, los que, en su conjunto, deben dar cuenta de los objetivos de una investigación (Mason y Merga, 2018).

La literatura que ha profundizado en la implementación de este formato identifica ventajas y riesgos. Entre las ventajas, se destaca que este formato de tesis permite a los y las estudiantes ahorrar tiempo y focalizar sus esfuerzos, ya que no deben escribir una tesis y en forma paralela o posteriormente publicar. Junto con eso, ayudaría a fortalecer el currículum de los doctores y doctoras recién titulados y los haría más competitivos para el mercado laboral en que se insertarán. Así también, al desarrollar la tesis en este formato, los y las estudiantes doctorales ganan experiencia en el oficio de publicar: aprenden a seleccionar revistas y a adaptarse a sus estilos y requisitos, se entrenan en el tipo de escritura académica que los artículos requieren, y sus productos reciben mayor retroalimentación, 
ya que a sus supervisores o supervisoras y comisiones evaluadoras se suma la revisión de pares de cada uno de los artículos que conforman el compendio (Paré, 2017; Guerin, 2016).

Entre los riesgos, se destaca que la fragmentación de las tesis en distintos artículos tensiona su unidad y coherencia, y así también su profundidad. Las TCP suponen la escritura de artículos breves, lo que entra en tensión con el objetivo de desarrollar la capacidad, por parte de los y las estudiantes, de construir marcos teóricos densos, trabajos de campos de largo aliento y de sostener un argumento consistente en un escrito de envergadura. Junto con eso, la publicación de artículos tiende a ser un proceso lento, que no depende del estudiante ni del programa doctoral que cursa, por lo que se corre el riesgo de exceder el tiempo estimado para el desarrollo de un doctorado. Así también, dada la novedad de este formato, impone nuevas exigencias tanto para los programas doctorales, los y las tutoras y estudiantes. Supone definir nuevos requisitos y criterios de evaluación y velar por su plausibilidad, nuevas formas de orientar y acompañar el proceso de desarrollo de la tesis y priorizar de manera distinta el tiempo y trabajo de los y las estudiantes (Mason y Merga, 2018; Paré, 2017; Peacock, 2017; Guerin, 2016).

Este tipo de tesis se han implementado preferentemente en el área de ciencias, tecnología, ingeniería, matemáticas y salud. En ciencias sociales y educación su desarrollo es más incipiente y particularmente desafiante para las investigaciones de carácter cualitativo. La tradición en investigación en ciencias sería más convergente con este formato de tesis, mientas que, en las ciencias sociales, tradicionalmente se habría privilegiado la publicación de libros por sobre artículos, altamente valorados en dichos campos del saber y comunidades. Junto con eso, la investigación cualitativa, de amplio desarrollo en ciencias sociales y en educación, tendría mayores dificultades para adaptarse a las TCP. Éstas suelen suponer un desarrollo teórico de mayor complejidad y trabajos de campo más dilatados, lo que hace más difícil su fragmentación en artículos independientes, la gestión de los tiempos de publicación y el resguardo de la coherencia. Estos elementos hacen que la implementación de la TCP en ciencias sociales y educación sean aún más desafiantes y más crítica la definición de requisitos y criterios de acompañamiento y evaluación, en orden a equilibrar la creciente competitividad del mercado académico con las particularidades de la producción de conocimiento en estas áreas del saber (Mason y Merga, 2018; de Rosa, 2016). 


\section{METODOLOGÍA DEL ESTUDIO}

Para cumplir con el propósito de identificar las ventajas y riesgos de la implementación de la opción de TCP en programas doctorales en ciencias sociales y en educación, se diseñó una estrategia cualitativa que contempló dos fases de recolección e interpretación de información. En la primera fase se recopiló, sistematizó y comparó la información disponible sobre los requisitos y características de 13 programas doctorales en ciencias sociales y educación. En la segunda, se realizaron cuatro entrevistas en profundidad a estudiantes de doctorado y egresadas de algunos de esos programas doctorales, que había desarrollado o están desarrollando sus tesis en esta modalidad.

En la primera etapa, se elaboró un listado de programas doctorales de referencia. Para eso se utilizaron dos criterios: primero, que fueran programas doctorales chilenos e internacionales en ciencias sociales o educación de reconocida calidad que contemplaran la opción de TCP; y segundo, que contaran con información pública sobre sus requisitos y características. Para establecer los doctorados de referencia, se realizó una consulta a 10 académicos pertenecientes al Doctorado en Educación de las universidades Alberto Hurtado y Diego Portales. Tras ese proceso, se estableció un listado preliminar con 23 programas doctorales de interés, ocho chilenos y 15 internacionales. Luego, se realizó un primer proceso de búsqueda, revisando la información disponible en línea sobre cada uno de los programas y verificando que esa información permitiese su sistematización en las categorías de interés: número de publicaciones que conforman el compendio; tipo de publicaciones (artículos, capítulos del libro y otros); estado de las publicaciones (publicadas, aceptadas o manuscritos); características de las revistas y/o libros; requisitos de autoría; y finalmente, estructura del compendio. Tras la aplicación de este segundo criterio se consolidó un listado con 13 programas doctorales en ciencias sociales y educación, cinco chilenos y ocho internacionales. 
Tabla 1 - Muestra de programas doctorales de referencia

\begin{tabular}{|l|l|}
\hline \multicolumn{2}{|l|}{ Chilenos } \\
\hline 1 & Doctorado en Educación, Pontificia Universidad Católica de Chile \\
\hline 2 & Doctorado en Psicología, Pontificia Universidad Católica de Chile \\
\hline 3 & Doctorado en Sociología, Pontificia Universidad Católica de Chile \\
\hline 4 & Doctorado en Psicología, Universidad Alberto Hurtado \\
\hline 5 & Doctorado en Psicología, Universidad Diego Portales \\
\hline & \\
\hline 6 & Doctorado en Educación y Sociedad, Universidad de Barcelona, España \\
\hline 7 & Doctorado en Educación, Universidad Autónoma de Barcelona, España \\
\hline 8 & Doctorado en Educación, Universidad Autónoma de Madrid, España \\
\hline 9 & Doctorado en Educación, Monash University, Australia \\
\hline 10 & Doctorado en Educación, University of Melbourne, Australia \\
\hline 11 & Doctorado en Educación, University of Cambridge, Reino Unido \\
\hline 12 & Doctorado en Política Educativa, Standford University, Estados Unidos \\
\hline 13 & Doctorado en Políticas y Liderazgo Educativo, University of Calfornia, Berkeley, Estados Unidos \\
\hline
\end{tabular}

Para el análisis de la información de esta primera etapa, se organizaron los datos de cada programa en cada una de las seis categorías. Luego, se hizo una comparación al interior de cada una de ellas. Finalmente, se interpretaron y describieron las principales tendencias por categoría, de acuerdo con un continuo entre dos polos, uno más estricto, con requisitos específicos y detallados, y uno más flexible, que presenta requisitos más generales y mayor apertura al criterio del o la candidata, de supervisores y supervisoras, o del programa doctoral.

En la segunda fase, se complementó el análisis anterior con entrevistas en profundidad a cuatro estudiantes o recién egresadas de distintos programas de la muestra, quienes estaban realizando o habían realizados sus tesis en la opción de compendio de publicaciones. Esto, con la intención de rescatar la experiencia y perspectivas de los y las estudiantes. Se les consultó sobre las ventajas y riesgo que, desde su experiencia, supone la realización de una TCP, así como sobre aspectos críticos que deben ser considerados al momento de diseñar e implementar esta opción de tesis. Las entrevistas fueron transcritas y luego analizadas en búsqueda de elementos comunes e información relevante para los propósitos de este artículo. 


\section{TENDENCIAS SOBRE REQUISITOS Y CARACTERÍSTICAS DE LAS TESIS POR COMPENDIO DE PUBLICACIONES}

A partir de la revisión y comparación de los requisitos y características de las TCP en los programas doctorales que conforman la muestra, fue posible establecer tendencias respecto al número y tipo de publicaciones exigidas, al estado de las publicaciones y su formato, a los requisitos de autoría y a las características de los compendios.

Con relación al número de publicaciones exigidas, se observa una relativa homogeneidad entre los programas: una clara mayoría plantea como requisito incluir tres publicaciones dentro de la tesis (8). Ahora bien, también existe un número menor de programas en que esto se deja a criterio del candidato y su supervisor (3).

Respecto al tipo de publicaciones, los artículos académicos son el formato preferente aceptado en los programas. Todos los programas de la muestra que presentan información al respecto aceptan o exigen artículos académicos. En esta categoría también se puede ubicar a los programas en dos polos: aquellos más estrictos, en que se aceptan solo artículos académicos (6) y aquellos más flexibles, en que se deja abierta la posibilidad de incluir otro tipo de publicaciones (4), preferentemente capítulos de libros, siempre y cuando cumplan con un criterio de calidad similar al de los artículos. En estos casos, la inclusión de otros tipos de trabajos requiere ser aprobada por el director o directora de tesis. Vale la pena mencionar también que incluso en este polo más flexible, suele pedirse un número mínimo de artículos académicos. En esta categoría, existe menor homogeneidad: el 60\% de los programas se ubica en el polo más estricto, es decir solo se aceptan artículos indexados, y un $40 \%$ en el polo más flexible.

Respecto al estado de las publicaciones, en el polo más estricto, algunos programas exigen que todos los trabajos estén ya publicados o aceptados para su publicación (4). En el polo más flexible, se permite incluir también trabajos que ya hayan sido enviados a revistas o bien manuscritos finales en un formato publicable (2). También, un número considerable de programas se encuentra en un punto intermedio, en que se pide al menos un artículo aceptado y se establecen algunos criterios para el resto (3). Ésta aparece como una categoría crítica que se relaciona con una de las desventajas que la literatura identifica para este formato de tesis: el tiempo que puede tomar el proceso de publicación.

Sobre las características de las revistas o libros en los que se debe publicar, si bien todos los programas plantean que las revistas y libros deben ser de reconocido prestigio, nuevamente es posible identificar polos en cuanto a especificidad y exigencia. En el polo más estricto, es requisito que las revistas 
estén indexadas en la colección principal de Web of Science o Scopus y dentro de los dos primeros cuartiles en cuanto al factor de impacto. En el polo más flexible, esto queda a criterio del supervisor. También existen programas que están en una posición intermedia dentro de este continuo, en que se indica que las revistas deben contar con proceso de revisión por pares y ser de prestigio dentro del área. En cuanto a las publicaciones en libros, se observa una tendencia similar. En el polo más estricto se encuentran los requisitos de dos programas: uno indica que la editorial de publicación debe ubicarse en una posición relevante según el sistema de Scholarly Publishers Indicators (SPI) y otro, que debe existir un proceso de revisión por pares similar al de las revistas. Por otro lado, en el polo más flexible, se encuentra un programa en que se indica simplemente que el trabajo debe ser publicado en una editorial de relevancia.

Con relación a los requisitos de autoría, en todos los programas con información al respecto, se observa como factor común el requisito de que el candidato sea autor principal de la mayoría de los artículos, lo que implica que más del 50\% del trabajo de la publicación sea responsabilidad suya. En el polo más estricto se pide que el candidato sea autor principal en todas las publicaciones (3), mientras que en el más flexible se establece un número mínimo de trabajos, que va desde uno a tres (6). En este polo más flexible se aceptan trabajos en que el candidato puede no ser el autor principal, siempre y cuando se declare específicamente su contribución y se mantenga al mínimo este tipo de trabajos. Cabe mencionar que ésta es una categoría en que se observa mayor especificidad en los requisitos, en la mayoría de los programas, incluso en aquellos que se ubican en el polo más flexible (por ejemplo, respecto al número máximo de coautores, o la autorización de los coautores para incluir la publicación como parte de la tesis, y así también, sobre la necesidad de una declaración explícita de la contribución del candidato).

Finalmente, respecto a la estructura del compendio, los programas coinciden en que se espera que el compendio logre articular el conjunto de publicaciones y que efectivamente dé cuenta de los objetivos del proyecto de investigación en el que se inscriben. Nuevamente, los programas más estrictos presentan requisitos más detallados y específicos. Dentro de estos, destaca el llamado modelo sándwich, que comienza con una introducción, seguida del compendio de publicaciones y finaliza con una conclusión. En los capítulos de introducción y conclusión se espera, por lo general, que se expliquen en mayor profundidad o extensión elementos tales como la metodología, el marco teórico y la revisión de literatura, la relación que existe entre las publicaciones y la contribución de la tesis al área de investigación. También algunos programas sugieren escribir secciones de transición entre una publicación y otra. En definitiva, 
si bien existen diferencias en cuanto al nivel de detalle en la información pública que presentan los programas, es posible identificar que todos van en una línea similar. Esta última categoría se relaciona con uno de los principales desafíos de las TCP, la necesidad de articular en un conjunto coherente la fragmentación que supone que los resultados de la investigación se presenten en publicaciones independientes.

\section{Tabla 2 - Tendencias de los requisitos y características de las TCP en los doctorados de la muestra}

\begin{tabular}{|c|c|c|c|}
\hline & $\begin{array}{c}\text { Polo estricto } \\
\text { Polo flexible }\end{array}$ & & Polo \\
\hline Número de publicaciones & $\begin{array}{c}3 \text { publicaciones } \\
(73 \%)\end{array}$ & A criterio del supervisor (27\%) \\
\hline Tipos de publicaciones & $\begin{array}{c}\text { Artículos académicos } \\
(60 \%)\end{array}$ & $\begin{array}{c}\text { Artículos académicos y } \\
\text { capítulos de libros } \\
(40 \%)\end{array}$ \\
\hline Estado de las publicaciones & $\begin{array}{c}\text { Todas publicadas o aceptadas } \\
(45 \%)\end{array}$ & $\begin{array}{c}\text { Al menos 1 publicada o } \\
\text { aceptada } \\
(33 \%)\end{array}$ & $\begin{array}{c}\text { Enviados o escritos en } \\
\text { formato publicable } \\
(22 \%)\end{array}$ \\
\hline Indexación & $\begin{array}{c}\text { WOS o Scopus, dos primeros } \\
\text { cuartiles de factor de impacto. } \\
(56 \%)\end{array}$ & $\begin{array}{c}\text { Revistas con revisión por } \\
\text { pares y de prestigio dentro } \\
\text { del área. } \\
(22 \%)\end{array}$ & $\begin{array}{c}\text { A criterio del supervisor } \\
(22 \%)\end{array}$ \\
\hline Autoría & $\begin{array}{c}\text { Autor principal en todas las } \\
\text { publicaciones } \\
(33 \%)\end{array}$ & $\begin{array}{c}\text { Autor principal en un número } \\
\text { mínimo de publicaciones. } \\
\text { Entre una y tres } \\
(67 \%)\end{array}$ \\
\hline Estructura del Compendio & $\begin{array}{c}\text { Definen en detalle capítulos y } \\
\text { sus componentes } \\
(73 \%)\end{array}$ & $\begin{array}{c}\text { Consideraciones generales } \\
\text { sobre sus componentes } \\
(27 \%)\end{array}$ \\
\hline
\end{tabular}

\section{LA EXPERIENCIA DE LAS ESTUDIANTES: VENTAJAS, RIESGOS Y ASPECTOS CRÍTICOS}

En cuanto a las ventajas y riesgos de esta modalidad desde la perspectiva de los y las estudiantes, las entrevistadas coinciden, en lo medular, con lo que señala la literatura. Por un lado, en cuanto a las ventajas de esta modalidad, sostienen que lo principal es ayudar al estudiante a insertarse en el mundo académico, porque se fortalece su currículum y porque permite conocer los estándares y dinámicas de la escritura de artículos para las revistas indexadas. Dicho de otro modo, la TCP se posiciona como una modalidad de tesis que prepararía de mejor manera a los estudiantes para el campo laboral de la academia y que favorece su inserción en ella con un currículum más competitivo. 
Por otro lado, en cuanto a los riesgos, las entrevistadas plantean que los estudiantes pueden tener dificultades para proporcionar una visión holística de su trabajo de tesis y que existe el riesgo de la fragmentación. Al mismo tiempo, se menciona que la necesidad de escribir textos breves y concisos, como el del formato de los artículos, puede limitar excesivamente el espacio para analizar la literatura de referencia y exponer la metodología de la investigación. Ahora bien, al momento de implementar las TCP estos riesgos pueden, a juicio de las entrevistadas, ser abordados a través de acciones específicas del programa de doctorado, como, por ejemplo, lineamientos claros sobre la estructura de la tesis y apoyo del programa y del supervisor o supervisora en su desarrollo.

Otro posible riesgo de las TCP, que adquiere relevancia en el ámbito de las ciencias sociales, y en el de la educación, es que este formato es más dúctil para investigaciones cuantitativas, ya que la producción de datos puede ser más expedita, y porque es más fácil dividir los resultados en diferentes artículos. Esto es consistente con lo encontrado en la revisión de literatura. Ahora bien, a pesar de esto, la entrevistadas plantean que cualquier investigación podría presentarse en esta modalidad, siempre y cuando esta decisión se tome en una fase inicial del proyecto y que las preguntas de investigación se delimiten correctamente, de manera de facilitar la producción de un artículo para cada una de ellas. En este sentido, nuevamente este riesgo se puede matizar a partir de lineamientos y acciones particulares.

Finalmente, de las respuestas de las entrevistadas, se destaca como un aspecto crítico a considerar al momento de diseñar e implementar esta opción de tesis, el valor que tienen los lineamientos que plantea el programa doctoral. En términos generales, las informantes destacan la importancia de generar consenso entre los y las profesoras del programa respecto a los estándares esperados para las TCP y prescribirlos de manera clara, ya que esto facilitaría la experiencia de los candidatos en su desarrollo. Además de lineamientos claros y específicos, las entrevistadas presentan otras acciones que podrían facilitar la experiencia de los candidatos. En ese marco, una entrevistada plantea la importancia de disponer de ejemplos concretos de TCP de alta calidad que puedan operar como modelos para guiar a los estudiantes. En esta misma línea, también se sugiere generar instancias grupales de apoyo y colaboración, en que, por un lado, se presenten a los estudiantes las distintas opciones de tesis y, por otro, se trabaje en torno al desarrollo del tipo de escritura académica que demandan los artículos indexados. Desde la experiencia de las entrevistadas, quizás lo más relevante, es contar con orientaciones claras sobre lo que se espera de una TCP que sirvan como guía para su desarrollo. 


\section{REFLEXIONES FINALES Y PROPUESTAS}

Como señala la literatura de referencia y las experiencias de quienes han optado por esta opción de tesis, la implementación de las TCP en doctorados en ciencias sociales y educación supone ventajas, como la experiencia que los y las estudiantes adquieren en la escritura de artículos científicos y el fortalecimiento de sus currículums y competitividad para su posterior inserción académica. Pero también supone riesgos: establecer requisitos de publicación demasiado estrictos o poco plausibles, dilatando así los tiempos de titulación; la poca ductilidad de este formato para investigaciones que requieren de desarrollos teóricos extensos o de trabajos de campo dilatado; y finalmente, el desafío de dotar de unidad y coherencia al compendio final, dada la fragmentación de la tesis en artículos independientes.

Al implementar las TCP es necesario reconocer estos riesgos y considerar maneras de preverlos o matizarlos, resguardando el equilibrio entre requisitos y exigencias, por una parte, con lineamientos claros, apoyos y espacios al criterio de estudiantes, supervisores y supervisoras, y de los propios programas, por otra. Desde esa perspectiva, al momento de implementar esta opción de tesis, nos parece relevante tomar en cuenta las siguientes siete consideraciones:

1. Criterios para optar por las TCP: Es recomendable que los programas doctorales ofrezcan las dos opciones para el desarrollo de las tesis, la opción monográfica y las TCP. La decisión de qué modalidad elegir para cada estudiante, es una decisión crítica para su proceso formativo. No debiese ser una decisión solo del o la estudiante, sino que debiese ser discutida y consensuada con el supervisor o supervisora, la respectiva comisión de la o el estudiante y/o la dirección de los programas. Para ello se debiese tener en cuenta la experiencia previa de las y los estudiantes en investigación y escritura de artículos, sus fortalezas y necesidades formativas y las características de la investigación que desea realizar (el tipo de desarrollo teórico que requiere, su diseño metodológicos y las características del trabajo de campo)

2. La necesidad de un diseño robusto: La etapa del diseño y fundamentación de las investigaciones, previo a la escritura de los artículos, se convierte en un momento de especial relevancia en el caso de las TCP. Es crucial que los estudiantes profundicen de manera temprana en la delimitación y fundamentación de sus problemas de investigación, así como en sus perspectivas teóricas y metodológicas. Esto permite que tengan claridad y fundamentos para diseñar objetivos susceptibles de ser desarrollados en artículos independientes, y así también, abre la posibilidad de anticipar una publicación de carácter teórico o metodológico. 
3. Apoyos a la escritura y publicación de artículos: La publicación de artículos requiere del desarrollo de una escritura académica particular, que no sólo remite al género argumentativo, sino que también responde a los estilos y requisitos de las revistas científicas indexadas. Desde esta perspectiva, es fundamental que los programas implementen dispositivos de apoyo a la escritura de artículos como, por ejemplo, talleres o cursos de escritura académica, tanto de la perspectiva lingüística como desde la experiencia en el oficio de publicar, espacios de lectura y comentarios entre docentes y estudiantes, y el aporte de buenos ejemplos, tanto de artículos como de compendios.

4. Equilibrar las exigencias de indexación con el diálogo con distintas comunidades y auditorios: El principal propósito de una tesis doctoral es producir conocimiento científico, para ser difundido preferentemente en circuitos académicos. Por ello es relevante que la modalidad de compendios exija la publicación de artículos indexado. Sin embargo, junto al nivel de indexación, es importante considerar los auditorios y comunidades para quienes se produce ese conocimiento. No siempre las revistas de altas exigencias de indexación coinciden con las publicaciones de mayor difusión en ciertos campos del conocimiento. Algo similar ocurre con los capítulos de libros, que en muchos casos, tiene mayor impacto en auditorios y comunidades específicas que los artículos indexados. Desde esta perspectiva, es importante que un estudiante doctoral desarrolle la capacidad de comunicar los resultados de sus investigaciones en distintos formatos y considerando distintos auditorios, y en consecuencia, que el compendio no se limite o restringa sólo a artículos de alta indexación.

5. Mantener el control de los tiempos de titulación: La exigencia de tres publicaciones aceptadas o publicadas, que contemplan los programas con requisitos más estrictos, pone en riesgo los tiempos de titulación oportuna y resta relevancia e influencia a las comisiones de evaluación. Desde esta perspectiva, parece razonable combinar artículos publicados o aceptados, para que él o la estudiante tenga la experiencia completa de publicar, con artículos manuscritos en condiciones de publicación. Una fórmula mixta de este tipo permite que tanto estudiantes como programas tengan control sobre los tiempos de titulación, y puedan o sean capaces de equilibrar entre el aporte de la evaluación de pares de los artículos publicados y aceptados, con la evaluación y acompañamiento por parte de las supervisoras o supervisores, comisiones y programas.

6. Combinar autoría individual con publicaciones colectivas: Tanto la responsabilidad individual sobre un artículo como la experiencia de la publicación colaborativa son aprendizajes deseables para un estudiante doctoral. En consecuencia, dentro de las tres publicaciones exigidas se debe idealmente considerar al menos una de autoría individual y una colectiva. 
7. Articulación y consistencia del compendio: Más allá del consenso entre los programas analizados sobre la necesidad de que el compendio considere una introducción y conclusión que articule las distintas publicaciones, es importante velar por la coherencia interna del conjunto -en oposición a la fragmentación que supone su organización en artículos-, que además permitan una explicación de mayor profundidad o extensión sobre la metodología, el marco teórico y la revisión de literatura, la relación que existe entre las publicaciones y la contribución de la tesis al campo de conocimiento en que se inserta la investigación.

\section{REFERÊNCIAS}

DE ROSA, A. S. (2016). Article, book format, or both? Shared criteria adopted for the double doctoral thesis format and language in a european/international joint networked phd program. En Inted2016: 10th international technology, education and development conference (pp. 1014-1026).

FARDELLA, C., Baleriola, E., y Enciso, G. (2020). Practices and Discourses of Academics: Local Lessons to Address the Digital Shift in Academic Management. Digital Education Review, (37), 64-78.

GUERIN, C. (2016). 'Connecting the dots: writing a doctoral thesis by publication', en C. Badenhorst \& C.Guerin (eds.), Research literacies and writing pedagogies for Masters and Doctoral writers, Brill, Leiden \& Boston, pp.31-50.

MASON, M.K. Merga y J.E. Morris (2019): Typical scope of time commitment and research outputs of ThesisbyPublicationin Australia,HigherEducation Research \& Development, 39(2), 244-258. DOI: 10.1080/07294360.2019.1674253

MASON, S., y Merga, M. (2018). Integrating publications in the social science doctoral thesis by publication. Higher Education Research \& Development, 37(7), 1454-1471.

O'KEEFFE, P. (2019): PhD by Publication: innovative approach to social science research, or operationalisation of the doctoral student ... or both?, Higher Education Research \& Development, 39(2), 288-301. DOI: 10.1080/07294360.2019.1666258 
PARÉ, A. (2017) Re-thinking the dissertation and doctoral supervision / Reflexiones sobre la tesis doctoral y su supervisión, Infancia y Aprendizaje, 40:3, 407-428, DOI: 10.1080/02103702.2017.1341102

PEACOCK, S. (2017). The PhD by publication. International Journal of Doctoral Studies, 12, 123-134. Recuperado de http://www.informingscience. org/Publications/3781

RIGBY, J., \& Jones, B. (2020). Bringing the doctoral thesis by published papers to the Social Sciences and the Humanities: A quantitative easing? A small study of doctoral thesis submission rules and practice in two disciplines in the UK. Scientometrics, 1-23.

\section{Renato Gazmuri Stein}

Doutor em Didática de História, Geografia e Ciências Sociais Universidade Autônoma de Barcelona). Professor associado da Faculdade de Educação da Universidade Diego Portales, Chile, onde é Diretor do Programa de Doutorado em Educação. E-mail: renato.gazmuri@udp.cl

\section{Alejandra Falabella Ambrosio}

Doctora en Sociología de la Educación del Institute of Education de la Universidad de Londres, Magíster en Antropología Social de la Universidad de Chile y Educadora de Párvulos de la Pontificia Universidad Católica de Chile. Académica asociada del Departamento de Política Educativa y Desarrollo Escolar de la Universidad Alberto Hurtado. Secretaria Académica Doctorado en Educación UAH/UDP.

\section{Tamara Canessa Aguilar}

Psicóloga, Pontificia Universidad Católica de Chile, Magíster en Educación, Universidad de Londres. 